\title{
LEUKEMIA CUTIS- A CASE REPORT
}

C. Nirmala ${ }^{1}$, Mangal V. Kulkarni²

1. Associate Professor, Department Of Pathology, Bangalore Medical College \&RI

2. Tutor, Department Of Pathology, Bangalore Medical College \&RI

\section{CORRESPONDING AUTHOR:}

Dr. C. Nirmala, No $85,17^{\text {th }}$ main, $15^{\text {th }}$ cross, Nanjundeshwara layout, J. P. Nagar $5^{\text {th }}$ phase, Bangalore 560078.

E-mail: chandrannirmala@yahoo.com

\section{HOW TO CITE THIS ARTICLE:}

C. Nirmala ${ }^{1}$, Mangal V. Kulkarni. "Leukemia Cutis - A Case Report". Journal of Evolution of Medical and Dental Sciences 2013; Vol2, Issue 25, June 24; Page: 4531-4535.

ABSTRACT: Leukemic cells infiltrating the dermis are referred to as leukemia cutis. Leukemia cutis is seen in myeloid and lymphoid leukemias. Granulocytic sarcoma presenting primarily as skin nodule [leukemia cutis] in CML is a rare entity. Presence of leukemia cutis points towards blast crisis and poorer prognosis even when the hematological parameters point towards a chronic phase. The authors report a case of Leukemia cutis in Chronic Myeloid Leukemia presenting with multiple large skin nodules all over the body. The clinicopathological features of this variant with FNAC cytology, skin biopsy and histopathology findings and peripheral blood smear are discussed with full details.

KEY WORDS; CML, Leukemia cutis, Granulocytic sarcoma (GS).

INTRODUCTION: Leukemic cells infiltrating the dermis are referred to as leukemia cutis. Leukemia cutis is seen in myeloid and lymphoid leukemias. Leukemia cutis is a rare presentation of Granulocytic sarcoma. Granulocytic sarcoma (chloroma) is formation of localized tumor mass in any part of the body, composed of granulocytic series of cells seen in myeloid leukemias. Granulocytic sarcoma is reported in 3-9\% cases of acute and chronic myelogenous leukemia (1). The authors report a case of Leukemia cutis in Chronic Myeloid Leukemia presenting with multiple large skin nodules all over the body. The clinicopathological features of this variant are discussed with full details.

CASE REPORT: A 33 yr old male presented with multiple cutaneous nodules over the face, back, anterior chest wall and limbs, associated with fever for duration of 3 months. The patient was referred from the dermatology department with a provisional diagnosis of leishmaniasis for FNAC of the skin nodules. On examination the skin nodules were of varying sizes ranging from 1-5 cms, largest measuring $5 \times 3 \mathrm{cms}$ and average size being $2 \times 1 \mathrm{cms}$. The nodules were present all over the body, more on face, back, chest wall and limbs. [Fig 1]The nodules were firm to hard and non tender on palpation. FNAC was performed at multiple sites from multiple nodules. Aspiration was 
performed by using $22 \mathrm{~g}$ needle and $10 \mathrm{ml}$ disposable syringe. The slides were air dried and wet fixed and stained with Giemsa and H\& E. FNAC smears showed presence of myeloid series of cells in varying stages of maturation composed of predominantly myelocytes, metamyelocytes, basophils and eosinophils. Blasts were upto 3\% and nucleated RBC's were 3-4\%. [fig 2] .A diagnosis of granulocytic sarcoma was suspected and subsequent complete haemogram, Bone marrow aspiration and biopsy of the skin nodule was performed.

Complete haemogram findings were Hemoglobin - 8.1gm/dl, Total Leucocyte count 3.30lakh/cumm, platelet count is $80,000 /$ cumm, differential leucocyte count blast:3\%, promyelocyte:5, myelocytes:24, metamyelocytes:30\% , eosinophils:09\%, basophils:04\%, neutrophils:25\%. The above peripheral smear finding is suggestive of chronic myeloid leukemia. Bone marrow study confirmed the feature of chronic myeloid leukemia [Fig 3].A skin biopsy of the nodule was performed. The histopathology showed dermis with a diffuse infiltrate of immature myeloid series of cells composed of myelocytes, metamyelocytes, eosinophils and neutrophils confirming the diagnosis of leukemia cutis [Fig 4\&5].

DISCUSSION: Leukemia cutis type of granulocytic sarcoma is a relatively rare condition and may manifest in various types of leukemia. In our present case we see leukemia cutis manifestation in a chronic myeloid leukemia. Leukemia cutis is reported in 2-8\% cases of CML (2).

Leukemia cutis is a rare presentation of granulocytic sarcoma .Granulocytic sarcoma (chloroma) is formation of localized tumor mass in any part of the body, composed of granulocytic series of cells seen in myeloid leukemias. GS is reported in 3-9\% cases of acute and chronic myelogenous leukemia.

GS was first coined by Rappaport, the other synonyms are chloroma, myeloblastoma, myelocytoma, extra medullary myeloid cell tumor (3).

GS is any extra medullary manifestation of acute myeloid leukemia and myeloproliferative diseases, (CML, essential thrombocythemia, polycythemia) myelodysplastic syndrome (1). GS can occur in any organ or tissue. The most common involvement is lymph node, small intestine, mediastinum, CNS, and orbit of eye and bone. (4) The most common involvement includes skull, paranasal sinuses, sternum, ribs, vertebrae and pelvis (3) .GS is reported in 3-9\% cases of acute and chronic myelogenous leukemia.

GS is more common in patients with AML M4 or M5 with Myeloblast which express T cell surface markers CD13 or CD14 and cytogenetic abnormalities of $t(8,21)$ or inv 16(1). Definitive diagnosis of GS requires a tissue biopsy. A panel of mono clonal antibodies against myeloperoxidase, CD68, CD43 and CD20 helps to accurately diagnose chloroma deposits. Presence of GS gives a poorer prognosis with poorer response to treatment and worse survival (1). GS occurring in a setting of MDS/MPD is equivalent to blast transformation.

Incidence of Leukemia cutis is more commonly seen in AML than that of CML. $90 \%$ of leukemia cutis may have other extramedullary involvement.(2)

Cutaneous lesions in leukemias can be of neoplastic and of paraneoplastic types. Leukemic infiltrates present as variety of skin lesions such as macules, papules, plaques, nodules and ulcers (5). Lesions can be erythematic or purpuric, presence of vesicles and bullae may represent unusual hypersensitivity reaction (2). In the present case the skin nodules were see as large firm nodules ranging from 1-5 cms and the largest nodule measuring $5 \times 3 \mathrm{cms}$. Cutaneous paraneoplastic diseases are a variety of inflammatory skin conditions that occur in leukemia patients. They include 
erythema nodosum, erythema annulare centrifugum, pyoderma gangrenosum, guttate psoriasis, vitiligo, stasis dermatitis and lesions mimicking urticaria.(2).leukemia cutis should be differentiated from sweet syndrome which is an acute febrile neutrophilic dermatosis found in patients of haematological malignancies (2).

On histopathology showed cutaneous leukemic infiltrate with display of several patterns. Most common is the interstitial infiltrate, others are the nodular infiltrate, dense band like infiltrate in the superficial dermis and sparse superficial, deep perivascular and peri adnexal infiltrate are occasional observed (6). The present case showed presence of diffuse leukemic infiltrate in the dermis.

A leukemic leukemia cutis is a condition where the extra medullary deposit in the skin occurs in patient with normal peripheral blood or bone marrow. In almost all cases acute leukemia developed shortly afterwards within 1-25 months.(1) Though the skin infiltrate show less than 5\% blast and reported in chronic phase of the disease, presence of leukemia cutis strongly suggests that blast crisis is imminent. (7) It is a more aggressive phase of disease (8). 90\% of leukemia cutis may have other extramedullary involvement. (2)

Histologically granulocytic sarcoma is classified on degree of maturation into three types, 1.Blastic, 2.Immature, 3. Differentiated. Blastic type is composed predominantly of myeloblasts, immature type is composed of myeloblasts and promyelocytes, differentiated type is composed of more promyelocytes and later stages of maturation(8). The present case predominantly mature neutrophils, myelocytes and metamyelocytes, eosinophilic myelocytes, very few blasts and promyelocytes , Hence categorized under differentiated type.

Granulocytic sarcoma presenting primarily as skin nodule [leukemia cutis] in CML is a rare entity. Presence of leukemia cutis points towards blast crisis and poorer prognosis even when the hematological parameters point towards a chronic phase. Clinical and morphological identification of GS remains a problem. Only by maintaining a high index of suspicion will clinician and pathologist recognize this tumor in majority of cases.

\section{REFERENCES:}

1. Wikipedia, myeloid sarcoma.

2. Manish singhal, Sarikal singh, Rajive kumar, Vinod raina. Extensive cutaneous manifestation: presenting feature of chronic myelocytic leukemia in second blast crisis. Indian J of Dermatol 2010; 55(3):265-7.

3. Jitender Mohan kunger, Mukesh Prasad Sharma H Talis. Granulocytic sarcoma of humerus, an unusual association of acute myeloblastic leukemia-a case report. Indian $\mathrm{j}$ pathol microbial 2009; (43):475-8.

4. U S Kanade, S D Birare, P Gadgil, SV swami. A case of chronic myelogenous leukemia cutis: a rare presentation. Indian J dermatol.2011; 56:451-2.

5. Yeon soo kang, Hai sung Kim ,Hyun Jeong park, Jun young lee et al. Clinical characteristics of 75 patients with leukemia cutis.J Korean med sci 2013;28:614-619

6. Gunnar wagner, Klaus kenchel, Walter back, Alina schulz et al. Leukemia cutisepidemiology, clinical presentation and differential diagnosis. JDDG 2012; 10:27-36

7. Richard.s.neiman, Maurice barcos, costan berard, Hugh bonner et al.Granulocytic sarcoma-a clinicopathological study of 61 biopsed cases. Cancer 1981; 48:1426-37.

Journal of Evolution of Medical and Dental Sciences/ Volume 2/ Issue 25/ June 24, 2013 Page 4533 


\section{CASE REPORT}

8. Elaine S Jaffe, Nancy lee Harris, Harald stein, James W Vardiman. World health organization classification of tumors, pathology and genetics of tumors of hematopoietic and lymphoid tissues. Myeloid sarcoma.2001; 104-5
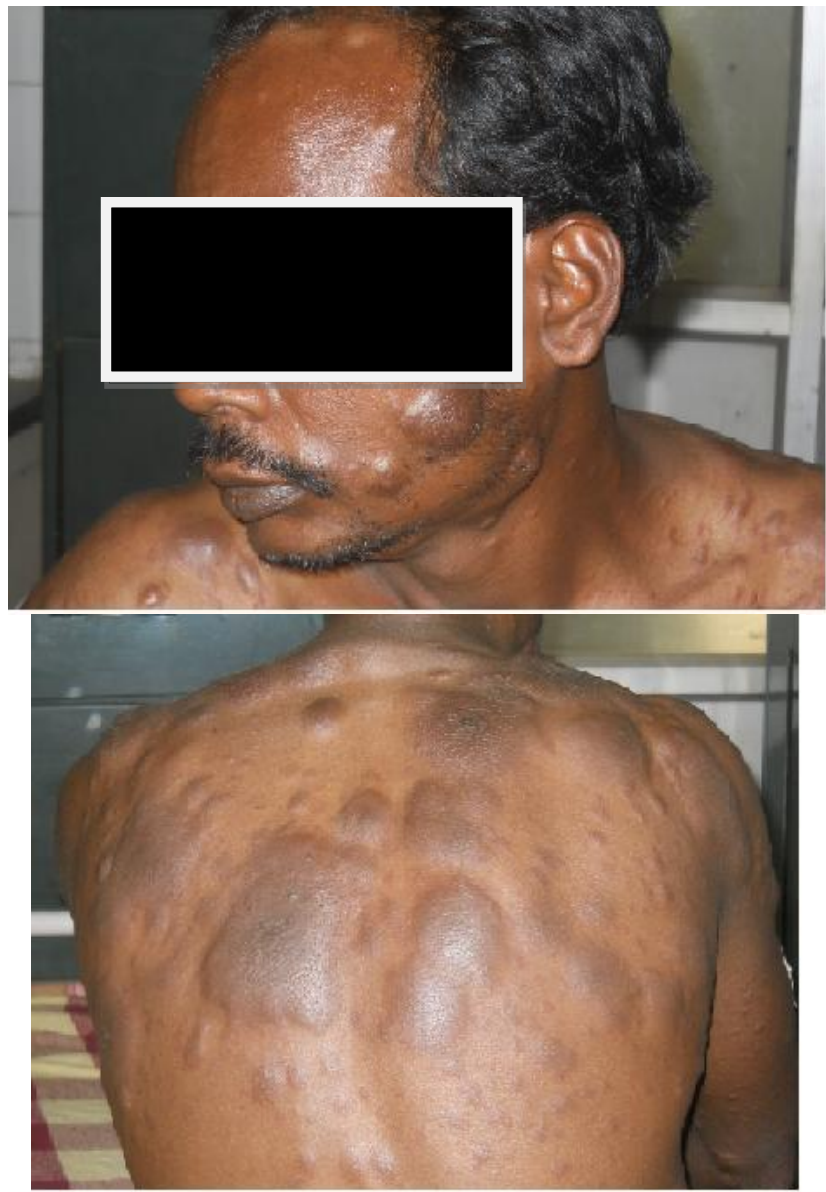

FIG .1 - Multiple skin nodules over face and back

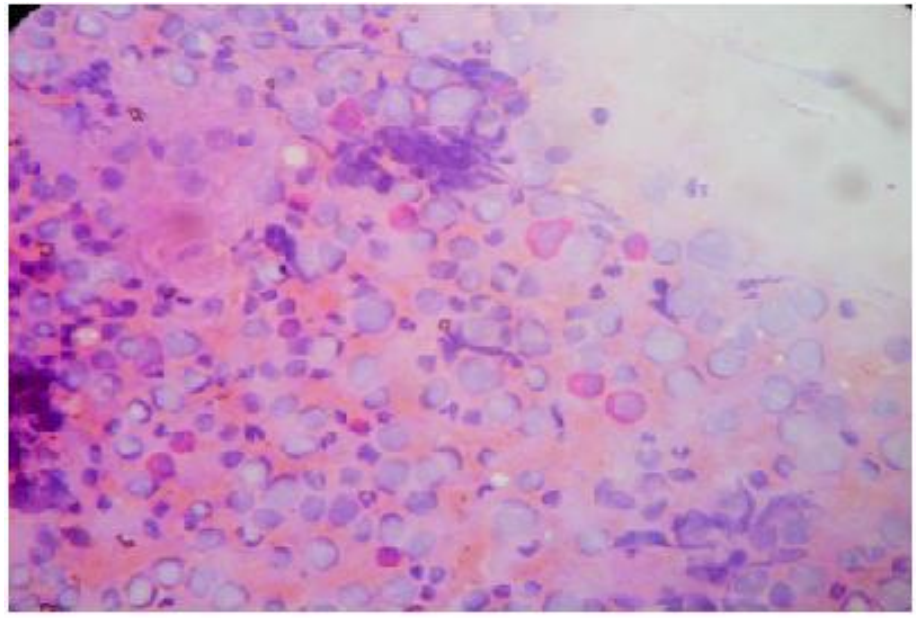

Fig 2- FNAC smears showing myeloid series of cells with eosinophilic myelocytes. H\&E stain 40X.

Journal of Evolution of Medical and Dental Sciences/ Volume 2/ Issue 25/ June 24, 2013 Page 4534 


\section{CASE REPORT}

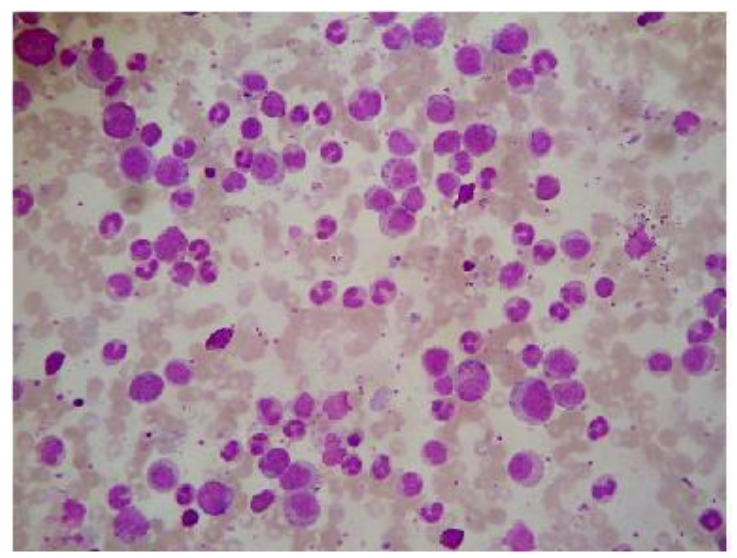

FIG .3 - Peripheral smear showing leucocytosis with immature myeloid series of cells composed predominantly of myelocytes, metamyelocytes, and neutrophils. Blasts 1-2\% suggesting Chronic Myeloid Leukemia. Leishman's stain, $40 \mathrm{X}$.

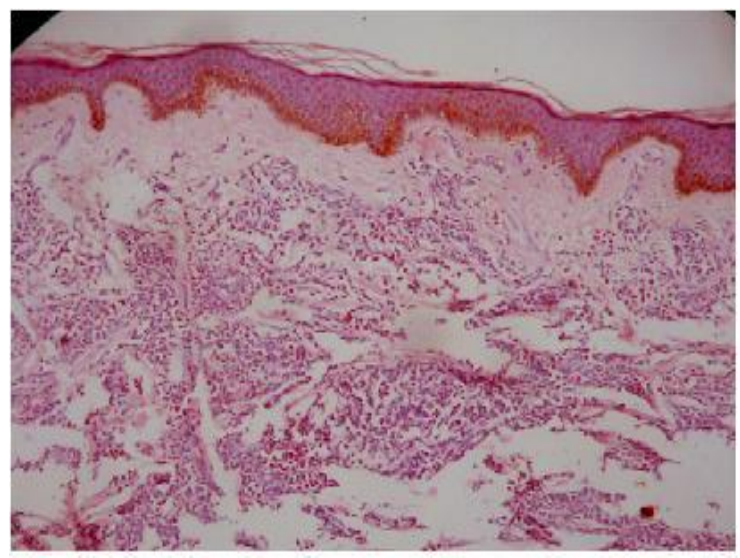

FIG 4 - Histopathology of skin biopsy, dermis showing diffuse pattern of infiltration by the leukemic cells. $\mathrm{H} \& \mathrm{E}, 10 x$.

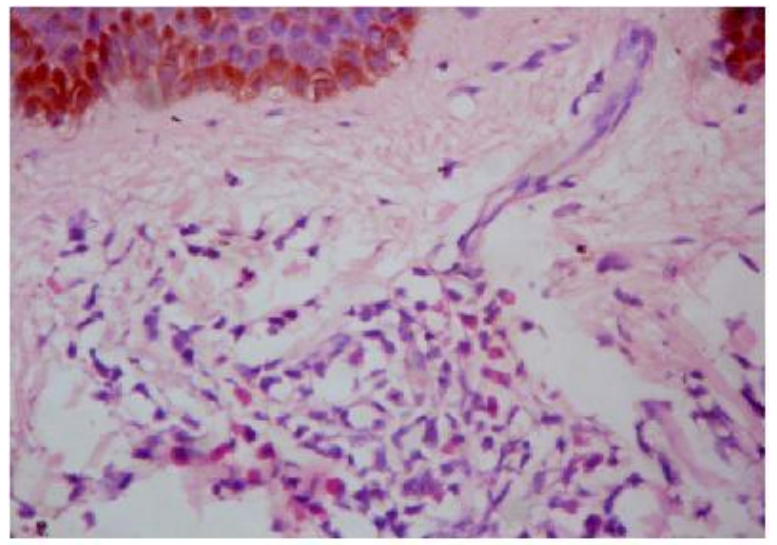

FIG.5 -Histopathology skin biopsy. Dermal infiltrates showing presence of eosinophilic myelocytes and other myeloid series of cells. $\mathrm{H} \& \mathrm{E}$ stain, $40 \mathrm{X}$. 